\title{
Development and validation of a simple HPLC assay method for ibuprofen, pseudoephedrine hydrochloride and chlorpheniramine maleate in syrup form
}

\author{
Hande Kazak Sarılmışer ${ }^{\odot *}$, N. Orçun Özçelik ${ }^{\odot}$, Banu Özkırım Arslan ${ }^{\circledR}$, \\ Mine Gökalp ${ }^{\odot}$, Arzu Ustaoğlu ${ }^{\odot}$, Udaya Kumar Dude ${ }^{\odot}$ and \\ Zdravka Knezevic
}

\author{
Abdi Ibrahim Pharmaceutical Company, Research and Development Center, Esenyurt, \\ Istanbul, Türkiye \\ (Received May 03, 2017; Revised October 26, 2017; Accepted October 27, 2017)
}

\begin{abstract}
A simple, rapid, sensitive, robust, stability-indicating HPLC analytical protocol was developed and validated for the analysis of Ibuprofen, Pseudoephedrine Hydrochloride, and Chlorpheniramine Maleate in syrup formulation. The samples were assayed by the Waters HPLC instrument using a GL Sciences Inertsil ODS4 column $(250 \mathrm{~mm}$ length $\times 4.6 \mathrm{~mm}$ width; $5 \mu \mathrm{m}$ particle size $)$ under timed gradient conditions. The flow rate of the mobile phase was $1.2 \mathrm{~mL} / \mathrm{min}$ and the eluent was monitored at $214 \mathrm{~nm}$. The method was validated by determining precision, linearity and range, specificity, accuracy and recovery, and robustness. The developed chromatographic method proved to be simple, precise, accurate, robust and reproducible. Moreover, the samples showed stability at room temperature over a period of $55 \mathrm{~h}$. Thus, this method would be employed for routine simultaneous quantification of Ibuprofen, Pseudoephedrine Hydrochloride, and Chlorpheniramine Maleate in Syrup formulation.
\end{abstract}

Keywords: HPLC; ibuprofen; pseudoephedrine hydrochloride; chlorpheniramine maleate; method validation (C) 2017 ACG Publications. All rights reserved.

\section{Introduction}

Ibuprofen (IBU) (Figure 1.1) is a non-steroidal anti-inflammatory drug that belongs to arylpropionic acid derivatives1 and is chemically (RS)-2-(4-(2-methylpropyl)phenyl)propanoic acid [1]. Pseudoephedrine hydrochloride (PSEH) (Figure 1.2), also known as $\{(+)$-threo- $\alpha-[1$-methylamino) ethyl] benzyl alcohol $\}$ hydrochloride, is a useful bronchodilator and nasal decongestant. Clinically, it shrinks swollen mucosa membranes, increases nasal airway passages, reduces nasal congestion, and diminishes tissue hyperemia [2]. Chlorpheniramine maleate (CHL), 3-(p-chlorophenyl)-3-(2-pyridyl)$\mathrm{N}, \mathrm{N}$-dimethyl propylamine (Figure 1.3) is a powerful first-generation alkyl amine antihistamine thatantagonises the H1-receptor and is widely used for symptomatic relief of the common cold and allergic rhinitis with weak sedative properties. The symptoms of allergic rhinitis include rash, watery

* Corresponding author E-Mail: hande.sarilmiser@abdiibrahim.com.tr 
HPLC assay method for ibuprofen, pseudoephedrine hydrochloride and chlorpheniramine maleate 62

eyes, itchy eyes and throat, cough, and sneezing. CHL is also effective against nausea and motion sickness, and its primary mechanism of action being is to reduce acetylcholine levels in the brain [3]. The combination of these drugs, is effective in cold, sinus and flu symptoms and used for upper respiratory infections [4]. Many analytical methods are described for determination of IBU, PSEH, and CHL in pharmaceuticals like spectrophotometer, gas chromatography and liquid chromatography with ion pair reagents.

The spectrophotometric method [5], liquid chromatography (HPLC) [6,7], and HPTLC [8-11] methods have been reported for the estimation of phenylephrine hydrochloride and chlorpheniramine maleate $[6,12]$ alone and in combination with other active pharmaceutical ingredients (API). Different from the literature, preservatives (Methyl Paraben-Na and Propyl Paraben-Na) and impurities (2-(4butylphenyl)propanoic acid, 2-(4-methylphenyl)propanoic acid, 2-[4-(2-methylpropanoyl)phenyl]propanoic acid, 2-(4-ethylphenyl)propanoic acid, and Ephedrine) beside the active ingredients can be detected and analyzed selectively by our assay method. The aim of our study was to develop and validate a simple, rapid, and selective HPLC method for simultaneous determination of IBU, PSEH, and CHL in a new combined pharmaceutical dosage form.<smiles>CC(C)Cc1ccc(C(C)C(=O)O)cc1</smiles>

1<smiles>CNC(C)C(O)c1ccccc1</smiles>

2<smiles>CN(C)CCC(c1ccc(Cl)cc1)c1ccccn1</smiles>

3

Figure 1. Structures of Ibuprofen (1), Pseudoephedrine hydrochloride (2) Chlorpheniramine maleate (3)

\section{Experimental}

\subsection{Materials}

IBU was purchased from Hubei Biocause Phamaceutical Co., Ltd. (China). PSEH was purchased from Malladi Drugs \& Pharmaceuticals Ltd. (India). CHL was purchased from Supriya Lifescience Ltd. (India). Standard information is shown in supplementary information. Decan-1sulfonic acid sodium salt, Potassium Dihydrogen Phosphate, and Triethylamine were purchased from Merck KGaA (Germany), Methanol and phosphoric acid were purchased from J.T.Baker (U.S.). All the chemicals used in the assays were HPLC grade.

\subsection{Apparatus and chromatographic conditions}

An HPLC system (Waters, USA) equipped with quaternary gradient pump, auto sampler, column oven and photodiode array detector (PDA) or UV detector was employed for analysis. A GL Sciences Inertsil ODS4 column $(250 \mathrm{~mm}$ length $\times 4.6 \mathrm{~mm}$ width; $5 \mu \mathrm{m}$ particle size $)$ was used for separation. Chromatographic data was acquired using Empower 2 software. Chromatographic separation was achieved using timed gradient. The mobile phase consisting of $\mathrm{A}: \mathrm{pH} 2.5$ buffer $(3.4 \mathrm{~g}$ $\mathrm{KH}_{2} \mathrm{PO}_{4}, 5 \mathrm{~g}$ Decan-1-sulfonic acid sodium salt, and $5 \mathrm{~mL}$ of Triethylamine in $1000 \mathrm{~mL}$ deionized water adjusted to $\mathrm{pH} 2.5$ with $\mathrm{H}_{3} \mathrm{PO}_{4}$ ) and $\mathrm{B}$ : Methanol (100\%). The gradient condition of the mobile phase is given in the supplementary information. The flow rate of the mobile phase was $1.2 \mathrm{~mL} / \mathrm{min}$ with detection at $214 \mathrm{~nm}$. The column temperature was $50^{\circ} \mathrm{C}$ and sample temperature was $25^{\circ} \mathrm{C}$. The injection volume was $20 \mu \mathrm{L}$. Diluent was Buffer : Methanol (800:200). 


\subsection{Diluent preparation}

$800 \mathrm{~mL}$ of $\mathrm{pH} 2.5$ buffer and $200 \mathrm{~mL}$ of methanol was mixed, filtered through $0.45 \mu \mathrm{m}$ membrane filter, and degassed.

\subsection{Standard solution preparation}

PSEH stock standard solution was prepared by transferring an accurately weighed $45 \mathrm{mg}$ of Pseudoephedrine hydrochloride working standard into a $50-\mathrm{mL}$ volumetric flask. $25 \mathrm{~mL}$ of diluent was added followed by sonication for $5 \mathrm{~min}$ using an ultrasonic source (TRANSSONIC-890). The solution was brought up to final volume with the diluent. CHL stock standard solution was prepared by transferring an accurately weighed $30 \mathrm{mg}$ of Chlorpheniramine maleate working standard into a 100$\mathrm{mL}$ volumetric flask. $10 \mathrm{~mL}$ of methanol was added followed by sonication for 5 min using an ultrasonic source. $40 \mathrm{~mL}$ of buffer was added and the solution was brought up to final volume with the diluent. For preparation of standard solution, accurately $30 \mathrm{mg}$ of Ibuprofen working standard was weighed and transferred into a $100-\mathrm{mL}$ volumetric flask. $10 \mathrm{~mL}$ of methanol was added followed by sonication for $5 \mathrm{~min}$ using an ultrasonic source. $5 \mathrm{~mL}$ of PSEH stock standard solution, $1 \mathrm{~mL}$ of CHL stock standard solution, and $40 \mathrm{~mL}$ of buffer were added and the solution was brought up to final volume with the diluent $\left(\mathrm{C}_{\mathrm{ibuprofen}}=0.3 \mathrm{mg} / \mathrm{mL}, \mathrm{C}_{\text {Pseudoephedrine } \mathrm{HCl}}=0.045 \mathrm{mg} / \mathrm{mL}, \mathrm{C}_{\text {Chlorphenamine Maleat }}=\right.$ $0.003 \mathrm{mg} / \mathrm{mL})$.

\subsection{Sample solution preparation}

Approximately $1.9 \mathrm{~g}$ syrup (equivalent to $\sim 1.5 \mathrm{~mL}, 30 \mathrm{mg}$ Ibuprofen, $4.5 \mathrm{mg}$ Pseudoephedrine $\mathrm{HCl}$, and $0.3 \mathrm{mg}$ Chlorpheniramine maleate) was weighed into a $100-\mathrm{mL}$ volumetric flask, dissolved with $50 \mathrm{~mL}$ of diluent and the solution was brought up to final volume with the diluent, filtered through $0.45 \mu \mathrm{m}$ PVDF filter and placed into HPLC vial $\left(C_{\text {ibuprofen }}=0.3 \mathrm{mg} / \mathrm{mL}\right.$, $\mathrm{C}_{\text {Pseudoephedrine } \mathrm{HCl}}=0.045 \mathrm{mg} / \mathrm{mL}, \mathrm{C}_{\text {Chlorphenamine Maleat }}=0.003 \mathrm{mg} / \mathrm{mL}$ ).

\subsection{Method validation}

\subsubsection{Precision}

Standard solution prepared at $100 \%$ working concentration was injected six times to evaluate the system precision. Peak areas obtained from injections and Relative Standard Deviation (RSD\%) value between them were calculated. Standard deviation (SD), confidence interval (CI 95\%) and peak performance parameters (symmetry factor, resolution, and theoretical plate number) were also observed. RSD\% between areas obtained from 6 consecutive injection of standard solution of no more than $2 \%$ was recommended. Beside system precision, repeatability (precision of the method) and intermediate precision were also evaluated. For repeatability, 2 standards and 6 syrup samples were prepared and the analysis was carried out. For intermediate precision, 6 assay samples were prepared and analyzed in a different day using a different device and column by a different analyst. In comparison of repeatability and intermediate precision results, the RSD values between result obtained from 12 samples for IBU, PSEH and CHL were calculated.

\subsubsection{Linearity and range}

To prove the linear response relation, peak areas of the standard solutions between $70 \%$ and $130 \%$ of specification at 6 different concentrations $(70 \%, 80 \%, 90 \%, 100 \%, 120 \%$ and $130 \%)$ were measured and linearity curves were plotted. After solutions were analyzed, $y=a x+b$ threshold was found and regression analysis was performed (y: area, a: slope, $b$ : intercept, $\mathrm{x}$ : concentration, $\mathrm{mg} / \mathrm{mL}$ ). Correlation coefficient between concentration and areas of more than 0.999 was sought. 


\subsubsection{Specificity}

Specificity test was performed to demonstrate the ability of the analytical method to measure only the intended substances in a given sample. Standard, unspiked sample, placebo, impurity standards, spiked sample, diluent and mobile phase were injected into the apparatus and analyzed. Sample solution was spiked with Impurity B (2-(4-butylphenyl)propanoic acid), Impurity D (2-(4methylphenyl)propanoic acid), Impurity $J$ (2-[4-(2-methylpropanoyl)phenyl]propanoic acid), Impurity $N$ (2-(4-ethylphenyl)propanoic acid), and Ephedrine. Spectrum of the injected solutions were taken. No peaks coming from diluent, placebo, and impurities were recommended at the retention time of IBU, PSEH, and CHL in standard, unspiked sample, and spiked sample chromatograms. IBU, PSEH, and CHL peaks in standard, unspiked sample, and spiked sample chromatograms are recommended not to interfere with other peaks. In chromatograms of standard, unspiked sample, and spiked sample solution, purity angle should be less than purity threshold for IBU, PSEH, and CHL peaks.

\subsubsection{Accuracy and recovery}

Accuracy of method was shown at 70\%, 100\% and 130\% levels by adding PSEH, CHL, and IBU raw materials to the placebo solution. In total, 9 samples were prepared ( 3 for each level) and injected twice. Recovery of each sample was recommended to be between $98.0 \%$ and $102.0 \%$. RSD\% between results obtained from 9 samples was recommended not to be more than $2.0 \%$.

\subsubsection{Robustness}

The robustness of the developed method was measured to evaluate the influence of deliberate variation in the chromatographic conditions. The robustness of the method was measured by changing the flow rate $(1.0$ and $1.4 \mathrm{~mL} / \mathrm{min})$, wavelength $(212 \mathrm{~nm}$ and $216 \mathrm{~nm})$, and column temperature $\left(48^{\circ} \mathrm{C}\right.$ and $52^{\circ} \mathrm{C}$ ). Robustness testing was performed in order to obtain information about those critical parameters affecting the response (peak area, retention time and found concentration).

\section{Results and discussion}

\subsection{Precision}

System precision was determined by performing injection repeatability test and the RSD values for PSEH, IBU, and CHL were found to be $0.09 \%, 0.15 \%$, and $0.39 \%$, respectively. RSD\% value between areas obtained from 6 consecutive injection of standard solution was less than $0.85 \%$. The low RSD values indicate that the system is precise. Also in comparison of repeatability and intermediate precision results, the RSD values between result obtained from 12 samples for PSEH, IBU, and CHL were found to be $0.26 \%, 0.22 \%$, and $0.39 \%$, respectively (data shown in supplementary information).

\subsection{Linearity and range}

For linearity evaluation, peak areas of the solutions prepared at 6 different concentration $(70 \%, 80 \%, 90 \%, 100 \%, 120 \%$, and $130 \%)$ were measured. The calibration curve for IBU was found to be linear in the range of $0.210-0.390 \mathrm{mg} / \mathrm{mL}$ with a correlation coefficient of 0.999 . The calibration curve for PSEH was found to be linear in the range of $0.032-0.045 \mathrm{mg} / \mathrm{mL}$ with a correlation coefficient of 0.999 . The calibration curve for CHL was found to be linear in the range of $0.0021-$ $0.0094 \mathrm{mg} / \mathrm{mL}$ with a correlation coefficient of 0.999 . Linearity figures are in the supplementary information. 


\subsection{Specificity}

The applied method demonstrated excellent specificity. There is no interfering with peaks in the chromatogram obtained from diluent, impurities and placebo. The spectrum of IBU, PSEH and CHL peaks did not interfere with the other peaks in Standard and Sample solution chromatograms. For the IBU, PSEH and CHL peaks, purity angle is less than purity threshold in Standard and Sample solution chromatograms. The selectivity of the method was proved. Blank and standard data are in the supplementary information. Spiked sample data is shown in Figure 2.

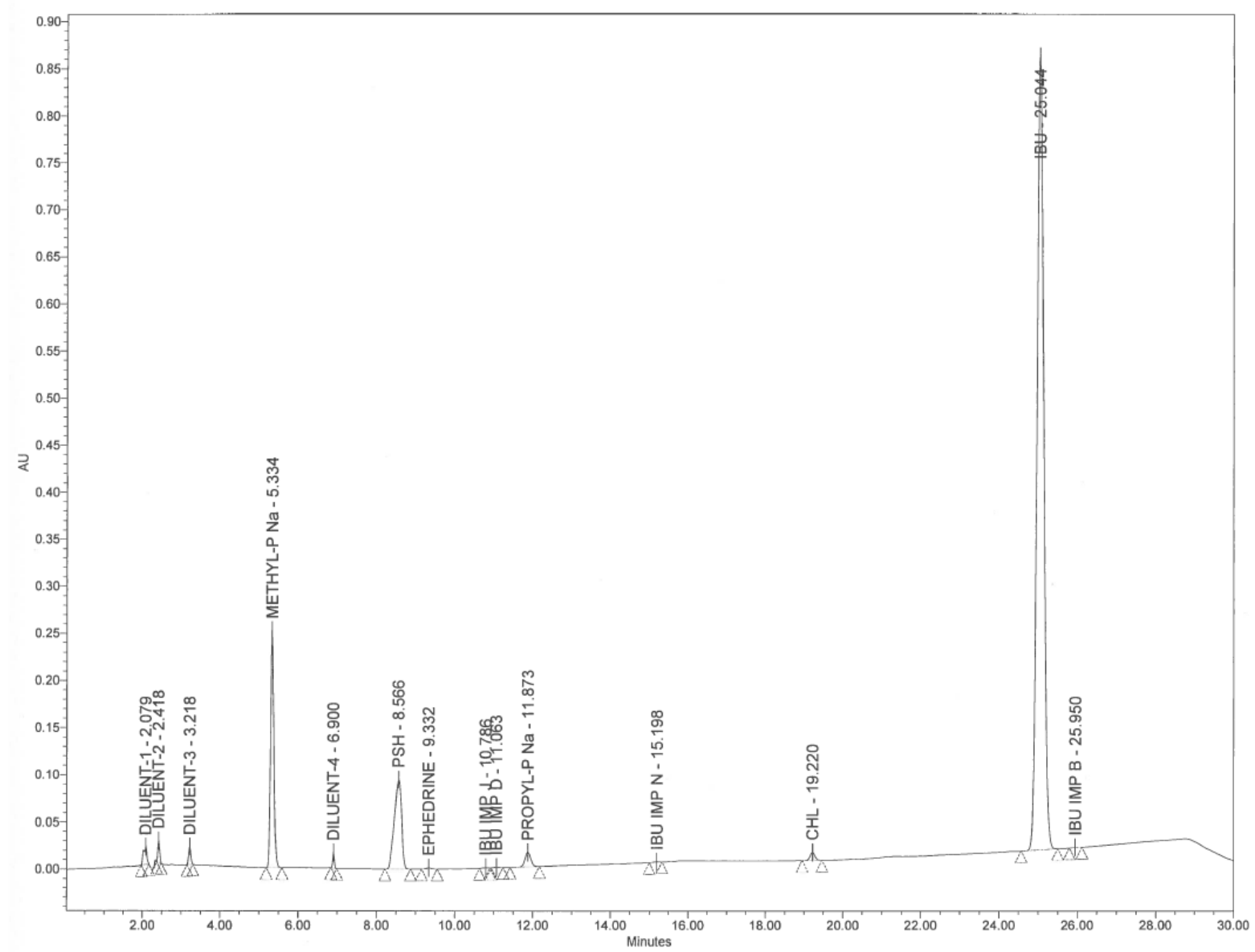

Figure 2. Chromatogram of Spiked Sample

\subsection{Accuracy and recovery}

Accuracy of the method was shown for $70 \%, 100 \%$ and $130 \%$ by adding IBU, PSEH, and CHL to placebo solution. 9 samples were prepared ( 3 for each level) and injected twice. Results are shown in Table 5. The bias correction values were found to be $0.000344,0.000012$, and 0.002089 for PSEH, CHL and IBU respectively by the accuracy profile. 
HPLC assay method for ibuprofen, pseudoephedrine hydrochloride and chlorpheniramine maleate 66

Table 5. Recovery data of validated method (\%)

\begin{tabular}{cccc}
\hline Solution Name & Recovery of IBU (1) & Recovery of PSEH (2) & Recovery of CHL (3) \\
\hline & 100.2 & 101.3 & 100.3 \\
$70 \%$ & 100.6 & 101.0 & 99.9 \\
& 100.6 & 101.2 & 100.8 \\
$100 \%$ & 100.1 & 101.1 & 100.5 \\
& 100.6 & 100.9 & 99.7 \\
$130 \%$ & 100.5 & 101.2 & 100.9 \\
& 99.0 & 99.7 & 100.2 \\
Average & 98.9 & 99.7 & 100.0 \\
SD & 98.9 & 99.6 & 100.5 \\
RSD\% & 99.9 & 100.6 & 100.3 \\
Confidence Interval (CI 95\%) & 0.77 & 0.74 & 0.40 \\
& 0.77 & 0.73 & 0.40 \\
& $99.9 \pm 0.5$ & $100.6 \pm 0.5$ & $100.3 \pm 0.3$
\end{tabular}

\subsection{Robustness}

With the Robustness parameter, the effect of changes in the method parameters and solution stability were examined. For this aim parameters which listed below were performed;

- Flow rate $\quad: 1.0 \mathrm{~mL} / \mathrm{min}$ and $1.4 \mathrm{~mL} / \mathrm{min}$

- Wavelength $\quad: 212 \mathrm{~nm}$ and $216 \mathrm{~nm}$

- Column Temperature : $48^{\circ} \mathrm{C}$ and $52^{\circ} \mathrm{C}$

Results showed that there was no significant change in Assay results and system suitability parameters for any components (data shown in supplementary information). The method is robust in view of changes to flow rate, wavelength and temperature.

For solution stability IBU, PSEH, and CHL standards and sample solution were prepared and analyzed at certain time intervals keeping storing conditions constant. Peak areas were recorded and similarity $\%$ was calculated. It was proved that standard solution is stable at $25^{\circ} \mathrm{C}$ for 56 hours and sample solution is stable at $25^{\circ} \mathrm{C}$ for 55 hours.

All of the related figures and tables for method validation are given in supporting information (Table S1-S11 and Figure S1-S5).

\section{Conclusions}

In this work, a simple and selective analytical method was successfully developed and validated for the detection and quantitation of Ibuprofen, Pseudoephedrine Hydrochloride, and Chlorpheniramine Maleate in syrup formulation. The method was demonstrated to be highly reproducible, specific, precise, and accurate. Because of the simplicity of the material and instruments used in this method, it can be applied in every laboratory equipped with a basic HPLC system. Furthermore, the HPLC method could also be suggested for the routine analysis of Ibuprofen, Pseudoephedrine Hydrochloride, and Chlorpheniramine Maleate in pharmaceutical dosage formulations.

\section{ORCID}

Hande Kazak Sarılmışer: 0000-0001-5896-5911

N. Orçun Özçelik: 0000-0003-0677-6179

Banu Özkırım Arslan: 0000-0002-7248-5794

Mine Gökalp: 0000-0002-7821-8232

Arzu Ustaoğlu: 0000-0002-8937-3553

Udaya Kumar Dude: 0000-0003-3660-1951

Zdravka Knezevic: 0000-0002-5759-8950 


\section{References}

[1] S. Jahan, J. Islam, R. Begum, R. Kayesh and A. Rahman (2014). A study of method development, validation, and forced degradation for simultaneous quantification of paracetamol and ibuprofen in pharmaceutical dosage form by RP-HPLC Method, Anal. Chem. Insigh. 9, 75-81.

[2] J.H. Ekpe, T. Tong and L. Rodriguez (2001). High-performance liquid chromatographic method development and validation for the simultaneous quantitation of naproxen sodium and pseudoephedrine hydrochloride impurities, J. Chromatogr. Sci. 39, 81-86.

[3] V. Jain and M.C. Sharma (2016). Validated RP-HPLC method for determining the levels of bromhexine $\mathrm{HCl}$, chlorpheniramine maleate, dextromethorphan $\mathrm{HBr}$ and guaiphenesin in their pharmaceutical dosage forms, J. Taibah Univ. Sci. 10, 38-45.

[4] B. Aşçı, Ö. Aksu Dönmez, A. Bozdoğan and S. Sungur in B. Şener (Ed.) (2009). Determination of Ibuprofen, Pseudoephedrine $\mathrm{HCl}$, Chlorpheniramine Maleate and Nipagen by Liquid Chromatography and Fractional Factorial Design (Innovations in Chemical Biology), Springer Netherlands, p.285-290.

[5] L.K. Soni, T. Narsinghani and C. Saxena (2011). Development and validation of UV-spectrophotometric assay protocol for simultaneous estimation of ebastine and phenylephrine hydrochloride in tablet dosage form using simultaneous equation method, Int. J. Chem. Tech. Res. 3, 1918-1925.

[6] M. Maithani, R. Raturi, G. Vertika and D. Kumar (2010). Development \& validation of RP-HPLC method for the determination of chlorpheniramine maleate and phenylephrine $\mathrm{HCl}$ in pharmaceutical dosage form, Int. Res. J. Pharm. 5,1-4.

[7] H. Senyuva and T. Ozden (2002). Simultaneous high-performance liquid chromatographic determination of paracetamol, phenylephrine $\mathrm{HCl}$, and chlorpheniramine maleate in pharmaceutical dosage forms, $J$. Chromatograph. Sci. 40, 97-100.

[8] S.S. Kardile, S.E. Potawale, S.S. Vidhate, A.M. Kashid, A.S. Bansode, H. Choudhury, T.L. Devale and P.D. Pawar (2015). Development and validation of HPTLC method for simultaneous estimation of ambroxol hydrochloride, phenylephrine hydrochloride, chlorpheniramine maleate, paracetamol and guaiphenesin in pharmaceutical formulation, J. Chem. Pharm. Res. 7(7), 169-177.

[9] N. Hunan and S. Multal (1986). Densitometric analysis of chlorpheniramine maleate, phenylephrine and acetaminophen by HPTLC method, Anal. Lett. 19, 7-8.

[10] P.V. Devarajan, M.H. Adani and A.S. Gandhi (2000). Simultaneous determination of lignocaine hydrochloride and phenylephrine hydrochloride by HPTLC, J. Pharmaceut. Biomed. Analys. 22, 685-690.

[11] I. Wouters, E. Roets and J. Hoogmartens (1984). Analysis of tablets containing acetylsalicylic acid and phenylephrine by high-performance liquid chromatography, J. Pharmaceut. Biomed. Analys. 2, 481-490.

[12] S. Rajurkar (2011). Simultaneous determination of chlorpheniramine maleate, paracetamol, pseudoephedrine $\mathrm{HCl}$ in pharmaceutical dosage form by HPLC method, Int. J. Life Sci. Pharma Res. 1, 94-100.

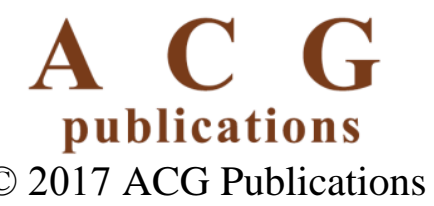

